\title{
Leapover lengths and first passage time statistics for Lévy flights
}

\author{
Tal Koren, ${ }^{1}$ Michael A. Lomholt, ${ }^{2}$ Aleksei V. Chechkin, ${ }^{3}$ Joseph Klafter,${ }^{1}$ and Ralf Metzler ${ }^{2}$ \\ ${ }^{1}$ School of Chemistry, Tel Aviv University, Tel Aviv 69978, Israel \\ ${ }^{2}$ Physics Department, Technical University Munich, 85748 Garching, Germany \\ ${ }^{3}$ Institute for Theoretical Physics NSC KIPT, Akademicheskaya st.1, 61108 Kharkov, Ukraine
}

\begin{abstract}
Exact results for the first passage time and leapover statistics of symmetric and one-sided Lévy flights (LFs) are derived. LFs with stable index $\alpha$ are shown to have leapover lengths, that are asymptotically power-law distributed with index $\alpha$ for one-sided LFs and, surprisingly, with index $\alpha / 2$ for symmetric LFs. The first passage time distribution scales like a power-law with index $1 / 2$ as required by the Sparre Andersen theorem for symmetric LFs, whereas one-sided LFs have a narrow distribution of first passage times. The exact analytic results are confirmed by extensive simulations.
\end{abstract}

PACS numbers: 02.50.Ey,05.40.Fb,89.65.Gh

The statistics of first passage times is a classical concept to quantify processes, in which it is of interest when the dynamic variable crosses a given threshold value for the first time, e.g., when a tracer in some aquifer reaches a certain probe position, two molecules meet to form a chemical bond, animals search for sparse food locations, or a share at the stock market crosses a preset market value [1, 2, 3]. Here, we revisit the first passage time problem for processes with non-trivial jump length distributions, namely, Lévy flights (LFs) and derive exact asymptotic expressions for the first passage time density $p_{f}(\tau)$ of symmetric and one-sided LFs. For the former, we obtain the Sparre Andersen universality $p_{f}(\tau) \simeq \tau^{-3 / 2}$, while a narrow behavior is found for onesided LFs. Apart from calculating the first passage times, we investigate the behavior of the first passage leapovers, that is, the distance the random walker overshoots the threshold value $d$ in a single jump (see Fig. 1). Surprisingly, for symmetric LFs with jump length distribution $\lambda(x) \simeq|x|^{-1-\alpha}(0<\alpha<2)$ the distribution of leapover lengths across $x=d$ is distributed like $p_{l}(\ell) \simeq \ell^{-1-\alpha / 2}$, i.e., it is much broader than the original jump length distribution. In contrast, for one-sided LFs the scaling of $p_{l}(\ell)$ bears the same index $\alpha$.

For processes subject to a narrow jump length distribution with finite second moment $\int_{-\infty}^{\infty} x^{2} \lambda(x) d x$ the crossing of a given threshold value $d$ is identical to the first arrival at $x=d[2]$. This is no longer true for LFs: Intuitively, a particle, whose jump lengths are distributed according to the symmetric long-tailed distribution $\lambda(x) \simeq|x|^{-1-\alpha}(0<\alpha<2)$ is likely to criss-cross the point $x=d$ multiple times before it eventually hits it, causing the first arrival at $d$ to be slower than its first passage across $d$ [4]. A measure for the ability to criss-cross $d$ is the distribution of leapover lengths, $p_{l}(\ell)$. Information on the leapover behavior is therefore important to the understanding of how far proteins searching for their specific binding site along DNA overshoot their target [5], climatic forcing visible in ice core records exceeds a given value [6], or to define better stock market strategies determining when to buy or sell a certain share

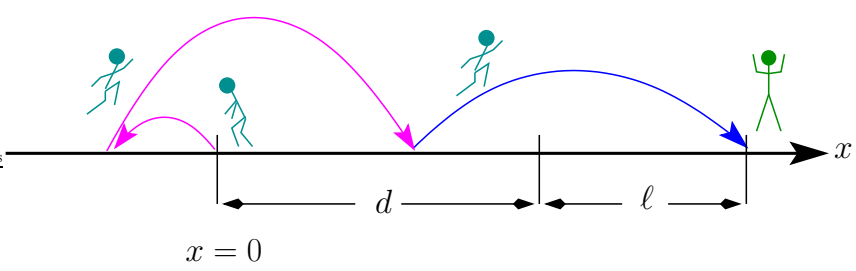

Figure 1: Schematic of the leapover problem: the random walker starts at $x=0$ and after a number of jumps crosses the point $x=d$, overshooting it by a distance $\ell$.

instead of fixing a threshold price [7]. The quantification of leapovers is vital to estimate how far diseases would spread once a carrier of that disease crosses a certain border [8]. Leapover statistics of one-sided LFs provide an interesting alternative interpretation of the distribution of the first waiting time in ageing continuous time random walks [9], just to name a few examples.

The master equation for a Markovian diffusion process,

$$
\begin{aligned}
\frac{\partial P(x, t)}{\partial t}=\frac{1}{\tau} \int_{-\infty}^{\infty} & {\left[\lambda\left(x-x^{\prime}\right) P\left(x^{\prime}, t\right)\right.} \\
& \left.-\lambda\left(x^{\prime}-x\right) P(x, t)\right] d x^{\prime}
\end{aligned}
$$

accounts for the influx of probability to position $x$, and the outflux away from $x$, where $\lambda(x)$ is a general, normalized jump length distribution. The time scale for single jumps is $\tau$. The solution to Eq. (11) in Fourier space is $P(k, t)=e^{-[1-\lambda(k)] t / \tau}$, denoting the Fourier transform $f(k)=\int_{-\infty}^{\infty} e^{i k x} f(x) d x$ by explicit dependence on the wave number $k$. For instance, for the symmetric jump length distribution $\lambda(x) \simeq \sigma^{\alpha}|x|^{-1-\alpha}$, one finds

$$
P(k, t)=e^{-K^{(\alpha)}|k|^{\alpha} t}
$$

with $K^{(\alpha)}=\sigma^{\alpha} / \tau$, the characteristic function of a symmetric Lévy stable law as obtained from continuous time random walk theory in the diffusion limit or from the equivalent space fractional diffusion equation [10].

In the following we study processes with the long-tailed composite jump length distribution

$$
\lambda(x) / \tau=\Theta(|x|-\varepsilon)\left[c_{1} \Theta(-x)+c_{2} \Theta(x)\right] /|x|^{1+\alpha},
$$


where $\Theta(x)$ is the Heaviside function. For $c_{1}=c_{2}, \lambda(x)$ defines a symmetric LF, and for $c_{1}=0$ and $c_{2}>0$ a completely asymmetric (one-sided) LF permitting exclusively forward jumps. The cutoff $\varepsilon$ excludes the singularity at $x=0$, but can be taken to be small, $\varepsilon \rightarrow 0$ [11].

In the theory of homogeneous random processes with independent jumps there exists a theorem, which provides an exact expression for the joint $\mathrm{PDF} p(\tau, \ell)$ of first passage time $\tau$ and leapover length $\ell(\ell \geq 0)$ across $x=d$ for a particle initially seeded at $x=0[12,13]$. We here evaluate this theorem, that appears to have been widely overlooked, and derive a number of new analytic results for $p_{f}(\tau)$ and $p_{l}(\ell)$ of symmetric and one-sided LFs. With the probability to jump longer than $x$,

$$
\mathcal{M}(x)=\int_{x}^{\infty} \lambda\left(x^{\prime}\right) d x^{\prime}, \quad x>0,
$$

the theorem states that the double Laplace transform of the joint PDF 12, 13]

$$
p(u, \mu)=\int_{0}^{\infty} \int_{0}^{\infty} e^{-u \tau-\mu \ell} p(\tau, \ell) d \tau d \ell
$$

is given in terms of the multiple integral

$$
\begin{aligned}
p(u, \mu)= & 1-q_{+}(u, d)-\frac{\mu}{u} \int_{0}^{d} \frac{\partial q_{+}(u, s)}{\partial s} d s \\
& \times \int_{-\infty}^{0} \frac{\partial q_{-}\left(u, s^{\prime}\right)}{\partial s^{\prime}} d s^{\prime} \int_{0}^{\infty} e^{-\mu s^{\prime \prime}} \\
& \times \mathcal{M}\left(d+s^{\prime \prime}-s^{\prime}-s\right) d s^{\prime \prime} .
\end{aligned}
$$

Here, we use the two auxiliary measures $q_{ \pm}(u, x)$ defined through Fourier transforms

$$
\begin{aligned}
& \tilde{q}_{ \pm}(u, k)=\int_{-\infty}^{\infty} e^{i k x} \frac{\partial q_{ \pm}(u, x)}{\partial x} d x \\
= & \exp \left\{ \pm \int_{0}^{\infty} \frac{e^{-u t}}{t} \int_{0}^{ \pm \infty}\left(e^{i k x}-1\right) P(x, t) d x d t\right\}
\end{aligned}
$$

and the condition $q_{ \pm}(u, 0)=0$. They are related to the cumulative distributions of the maximum, $Q_{+}(t, d)=$ $\operatorname{Pr}\left\{\max _{0 \leq \tau \leq t} x(\tau)<d\right\}$, and minimum, $Q_{-}(t, d)=$ $\operatorname{Pr}\left\{\min _{0 \leq \tau \leq t} x(\tau)<d\right\}$, of the position $x(t)$ such that $q_{ \pm}(u, d)=u \int_{0}^{\infty} e^{-u t} Q_{ \pm}(t, d) d t$. The complicated integrals above reduce to elegant results for symmetric and one-sided LFs, as we show now.

For symmetric LFs $\left(c_{1}=c_{2} \equiv c\right)$, the propagator is defined by the characteristic function (2) with generalized diffusion coefficient $K^{(\alpha)}=2 c \Gamma(1-\alpha) \cos (\pi \alpha / 2) / \alpha$. In the limit $u \rightarrow 0$ (long time limit), we obtain from Eq. (7)

$$
\tilde{q}_{+}(u, k) \sim \frac{u^{1 / 2}}{\sqrt{K^{(\alpha)}}|k|^{\alpha / 2}} \exp \left\{\frac{i \operatorname{sign}(k) \pi \alpha}{4}\right\} .
$$

Inverse Fourier transform yields

$$
q_{+}(u, d) \sim \frac{2 u^{1 / 2}}{\alpha \sqrt{K^{(\alpha)}} \Gamma(\alpha / 2)} d^{\alpha / 2}, \quad d>0
$$

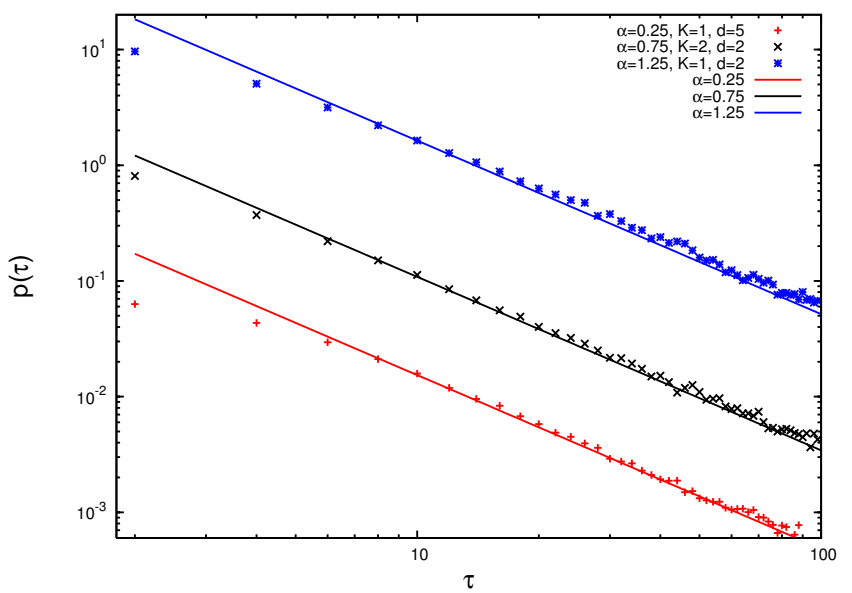

Figure 2: First passage time density $p_{f}(\tau)$ for symmetric LFs with Sparre Andersen universality $p_{f}(\tau) \simeq \tau^{-3 / 2}$. The curves for $\alpha=0.75$ and 1.25 are multiplied by a factor 10 and 100, for better visibility. Lines: theory. Symbols: simulations.

such that from $p_{f}(u)=1-q_{+}(u, d)$ we find

$$
p_{f}(\tau) \sim \frac{d^{\alpha / 2}}{\alpha \sqrt{\pi K^{(\alpha)}} \Gamma(\alpha / 2)} \tau^{-3 / 2} .
$$

This is the exact asymptotic first passage time PDF of symmetric LFs. Fig. 2 demonstrates good agreement with simulations results, for which the algorithm from Ref. 14] was used to obtain random numbers distributed according to Lévy stable laws. We note that previously only the $\tau^{-3 / 2}$ scaling was known from simulations and application of the Sparre Andersen theorem [4].

For symmetric LFs, for $0<\alpha<2$ we obtain that

$$
\mathcal{M}(x)=\frac{K^{(\alpha)}}{2 \Gamma(1-\alpha) \cos (\pi \alpha / 2)} x^{-\alpha}, \quad x>0 .
$$

Using that for symmetric LFs $q_{-}(\tau, x)=q_{+}(\tau,-x)$ it turns out after some transformations from Eq. (6) that

$$
p_{l}(\mu)=\int_{0}^{\infty} e^{-\mu \ell} \frac{\sin (\pi \alpha / 2)}{\pi} \frac{(d / \ell)^{\alpha / 2}}{d+\ell} d \ell
$$

from which it follows immediately that

$$
p_{l}(\ell)=\frac{\sin (\pi \alpha / 2)}{\pi} \frac{d^{\alpha / 2}}{\ell^{\alpha / 2}(d+\ell)},
$$

see Fig. 3. Note that $p_{l}$ is normalized. In the limit $\alpha \rightarrow 2$, $p_{l}(\ell)$ tends to zero if $\ell \neq 0$ and to infinity at $\ell=0$ corresponding to the absence of leapovers in the Gaussian continuum limit. However, for $0<\alpha<2$ the leapover PDF follows an asymptotic power-law with index $\alpha / 2$, and is thus broader than the original jump length PDF $\lambda(x)$ with index $\alpha$. This is a remarkable finding: while $\lambda$ for $1<\alpha<2$ has a finite characteristic length $\langle|x|\rangle$, the mean leapover length diverges. 


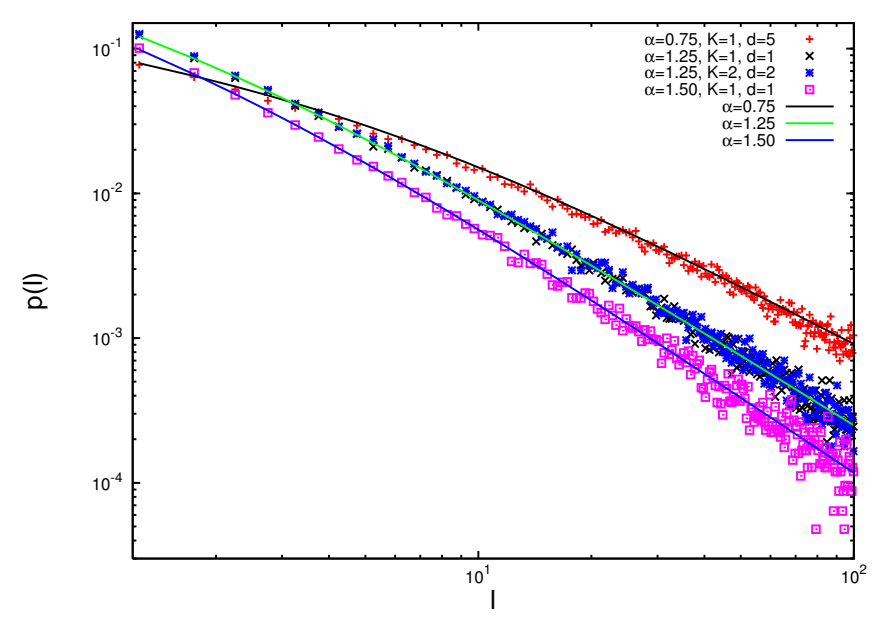

Figure 3: Leapover density $p_{l}(\ell)$ for symmetric LFs.

Consider now one-sided LFs with $c_{1}=0$ in Eq. (3). In this case, the PDF has the characteristic function

$$
P(k, t)=\exp \left\{-K^{(\alpha)} t|k|^{\alpha}\left[1-i \operatorname{sign}(k) \tan \left(\frac{\pi \alpha}{2}\right)\right]\right\}
$$

where $K^{(\alpha)}=c_{2} \Gamma(1-\alpha) \cos (\pi \alpha / 2) / \alpha$ and $\mathcal{M}(x)$ for $x>$ 0 is twice the expression in Eq. (11). Eq. (7) leads to

$$
\tilde{q}_{+}(u, k)=\frac{u}{u+\zeta}, \zeta=K^{(\alpha)}(-i k)^{\alpha} / \cos \left(\frac{\pi \alpha}{2}\right),
$$

as $(-i k)^{\alpha}=[-i \operatorname{sign}(k)|k|]^{\alpha}=|k|^{\alpha} \exp [-i \operatorname{sign}(k) \pi \alpha / 2]$. From this we calculate that the Fourier transform of $\langle\exp (-u \tau)\rangle=\int_{0}^{\infty} \exp (-u \tau) p_{f}(\tau) d \tau$ can be written as

$$
\int_{-\infty}^{\infty} e^{i k x}\left\langle e^{-u \tau}\right\rangle d x=\frac{(-i k)^{\alpha-1}}{(-i k)^{\alpha}+u \cos (\pi \alpha / 2) / K^{(\alpha)}}
$$

and we change the variable $i k \rightarrow-s$ to find [15]

$$
\left\langle e^{-u \tau}\right\rangle=E_{\alpha}\left[-\frac{u}{K^{(\alpha)}} \cos \left(\frac{\pi \alpha}{2}\right) d^{\alpha}\right] .
$$

Here, we used the definition of the Mittag-Leffler function

$$
\int_{0}^{\infty} E_{\alpha}\left(-\theta x^{\alpha}\right) e^{-s x} d x=\frac{s^{\alpha-1}}{s^{\alpha}+\theta} .
$$

whose series expansion and asymptotic behavior are [10]

$$
E_{\alpha}(-z)=\sum_{n=0}^{\infty} \frac{(-z)^{n}}{\Gamma(1+\alpha n)} \sim \sum_{n=0}^{\infty} \frac{(-1)^{n} z^{-1-n}}{\Gamma(1-\alpha[n+1])} .
$$

From the relation between $E_{\alpha}$ and the $M_{\alpha}$-function [16],

$$
\int_{0}^{\infty} e^{-u t} M_{\alpha}(t) d t=E_{\alpha}(-u), 0<\alpha<1,
$$

the following result for the first passage time PDF yields

$$
p_{f}(\tau)=\frac{K^{(\alpha)}}{\cos (\alpha \pi / 2) d^{\alpha}} M_{\alpha}\left(\frac{K^{(\alpha)} \tau}{\cos (\alpha \pi / 2) d^{\alpha}}\right) .
$$

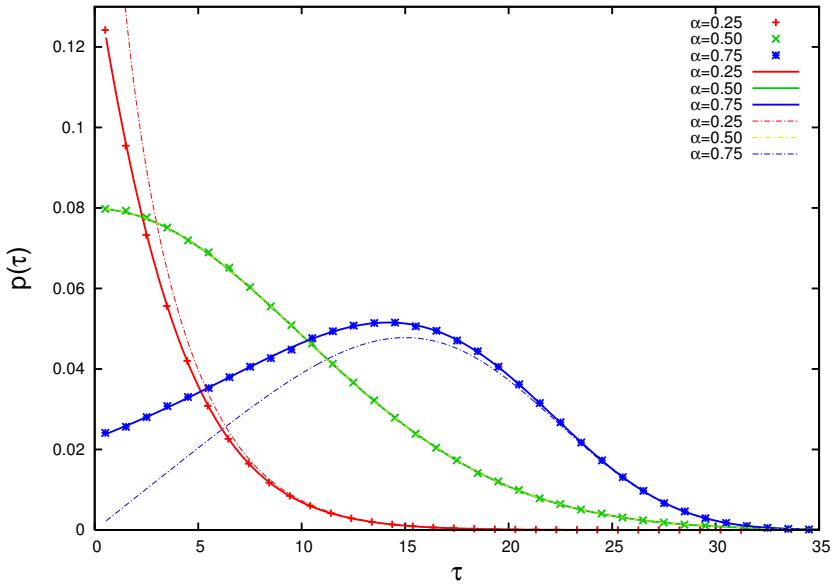

Figure 4: First passage density for one-sided $\operatorname{LF}\left(K^{(\alpha)}=1\right)$. The thick lines represent numerical evaluations of the exact analytic expression, while the thin dashed lines correspond to the asymptotic behavior (23). Symbols: simulations.

The $M_{\alpha}$-function has the series representation and asymptotic behavior with exponential decay

$$
\begin{aligned}
& M_{\alpha}(z)=\sum_{n=0}^{\infty} \frac{(-z)^{n}}{n ! \Gamma(1-\alpha-\alpha n)} \\
& \quad \sim \frac{(\alpha z)^{(\alpha-1 / 2) /(1-\alpha)}}{\sqrt{2 \pi(1-\alpha)}} \exp \left[-\frac{1-\alpha}{\alpha}(\alpha z)^{1 /(1-\alpha)}\right] .
\end{aligned}
$$

The moments of the $M_{\alpha}$-function are obtained through

$$
\int_{0}^{\infty} z^{n} M_{\alpha}(z) d z=\lim _{s \rightarrow 0}(-1)^{n} \frac{d^{n}}{d s^{n}} E_{\alpha}(-s)=\frac{\Gamma(n+1)}{\Gamma(1+\alpha n)},
$$

from which we calculate the mean first passage time

$$
\langle\tau\rangle=\frac{d^{\alpha} \cos (\pi \alpha / 2)}{K^{(\alpha)} \Gamma(1+\alpha)}
$$

that is finite and grows with the $\alpha$ th power of the distance $d$. For $\alpha=1 / 2$, we recover the exact form [17]

$$
p_{f}(\tau)=K^{(\alpha)} \sqrt{\frac{2}{\pi d}} \exp \left(-\frac{\left(K^{(\alpha)}\right)^{2} \tau^{2}}{2 d}\right) .
$$

The first passage $\mathrm{PDF} p_{f}(\tau)$ is displayed in Fig. 4 in nice agreement with the simulations. Note that for $\alpha \leq 1 / 2$ the tail of $\lambda(x)$ is so long that it is most likely to cross $x=d$ in the first jump, while for $\alpha>1 / 2, p_{f}(\tau)$ has a maximim at finite $\tau>0$.

To obtain the leapover statistics for the one-sided LF, we first note that since $P(x<0, t)=0$ (only forward steps are permitted) we have $q_{-}(u, k)=1$, and thus $\partial q_{-}(u, x) / \partial x=\delta(x)$. Combining Eqs. (6) and (7),

$$
\begin{array}{r}
\left\langle e^{-\mu \ell}\right\rangle=1-\lim _{u \rightarrow 0} \frac{\mu}{u} \int_{0}^{d} \int_{0}^{\infty} e^{-\mu s^{\prime}} \mathcal{M}\left(d+s^{\prime}-s\right) \\
\times \frac{\partial q_{+}(u, s)}{\partial s} d s^{\prime} d s .
\end{array}
$$




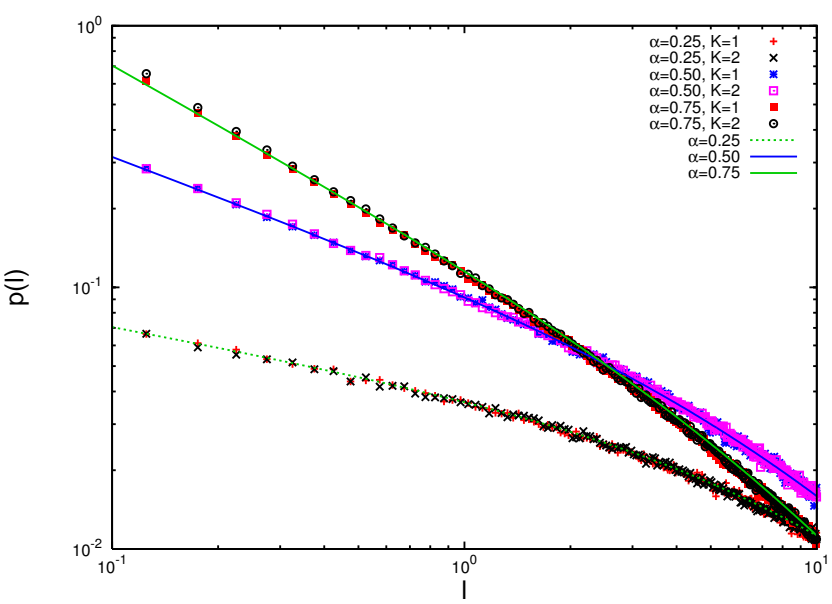

Figure 5: Leapover distribution for one-sided LF with $d=10$.

With the small $u$ expansion of the Mittag-Leffler function, Eqs. (17) and (19) produce

$$
\frac{\partial q_{+}(u, x)}{\partial x}=\frac{u \cos (\pi \alpha / 2)}{K^{(\alpha)} \Gamma(\alpha)} x^{\alpha-1} .
$$

Eqs. (15) and (28) inserted into Eq. (27) then yield

$$
p_{l}(\mu)=\left\langle e^{-\mu \ell}\right\rangle=\frac{\sin (\pi \alpha)}{\pi} \int_{0}^{\infty} e^{-\mu \ell} \frac{d^{\alpha}}{\ell^{\alpha}(d+\ell)},
$$

leading to the leapover PDF

$$
p_{l}(\ell)=\frac{\sin (\pi \alpha)}{\pi} \frac{d^{\alpha}}{\ell^{\alpha}(d+\ell)},
$$

which corresponds to the result obtained in Ref. 17] from a different method. Thus, for the one-sided LF, the scaling of the leapover is exactly the same as for the jump length distribution, namely, with exponent $\alpha$.

The leapover distribution (30) also provides a new aspect to the first waiting time in a renewal process with broad waiting time distribution $\psi(t) \simeq t^{-1-\beta}(0<\beta<$ 1). Interprete the position $x$ as time and the jump lengths drawn from the one-sided $\lambda(x)$ as waiting times $t$. Consider an experiment, starting at time $t_{0}$, on a system prepared at time 0 (corresponding to position $x=0$ ). Then the first recorded waiting time $t_{1}$ of the system will be distributed like $p_{1}\left(t_{1}\right)=\pi^{-1} \sin (\pi \alpha) t_{0}^{\alpha} /\left[t_{1}^{\alpha}\left(t_{0}+t_{1}\right)\right]$, as obtained from a different reasoning in Ref. [9]. We note that the first passage time $\tau$ in this analogy corresponds to the number of waiting events.

While for symmetric LFs it was previously established that the first passage time distribution follows the universal Sparre Andersen asymptotics $p_{f}(\tau) \simeq \tau^{-3 / 2}$, here we derived the prefactor of this law, in particular, its dependence on the generalized diffusion coefficient $K^{(\alpha)}$.
For the same case, we derived the leapover distribution $p_{l}(\ell)$, that is surprising for two reasons: first, $p_{l}(\ell)$ is independent of $K^{(\alpha)}$, synonymous to the noise strength; and second, its power-law exponent is $\alpha / 2$, and thus $p_{l}(\ell)$ is broader than the original jump length distribution.

For one-sided LFs, we recovered the previously reported leapover distribution and derived the so far unknown first passage time distribution. While the leapovers follow the same asymptotic scaling $p_{l}(\ell) \simeq$ $\ell^{-1-\alpha}$ as the jump lengths $\lambda(x)$, once more independent of $K^{(\alpha)}$, the first passage times are narrowly distributed. We also drew an analogy between the leapovers and the first waiting time in a subdiffusive renewal process. For both symmetric and one-sided LFs, extensive simulations showed nice agreement with the theoretical results, without adjustable parameters.

We acknowledge partial funding from NSERC and the Canada Research Chairs programme.

[1] S. Redner, A Guide to First-Passage Processes (Cambridge University Press, Cambridge, 2001).

[2] B. D. Hughes, Random Walks and Random Environments, Vol. 1 (Oxford University Press, Oxford, 1995).

[3] N. G. van Kampen, Stochastic Processes in Physics and Chemistry (North-Holland, Amsterdam, 1981).

[4] A.V. Chechkin et al., J. Phys. A 36, L537 (2003).

[5] M. A. Lomholt, T. Ambjörnsson, and R. Metzler, Phys. Rev. Lett. 95, 260603 (2005).

[6] P. D. Ditlevsen, Geophys. Res. Lett. 26, 1441 (1999).

[7] J.-P. Bouchaud and M. Potters, Theory of Financial Risk and Derivative Pricing: From Statistical Physics to Risk Management (Cambridge University Press, Cambridge, 2004).

[8] D. Brockmann, L. Hufnagel, and T. Geisel, Nature 439, 462 (2006); L. Hufnagel, D. Brockmann, and T. Geisel, Proc. Natl. Acad. Sci. 101, 15124 (2004).

[9] E. Barkai, Phys. Rev. Lett. 90, 104101 (2003).

[10] R. Metzler and J. Klafter, J. Phys. A 37, R161 (2004); Phys. Rep. 339, 1 (2000).

[11] The same trick is used in computer simulations of LFs. In a mathematical sense, this means that $\tau \rightarrow 0$ such that $u \tau \ll 1$ is fulfilled.

[12] A. V. Skorokhod, Random Processes with Independent Increments (Nauka, Moscow, 1964), in Russian.

[13] I. I. Gikhman and A. V. Skorohod, Theory of Stochastic Processes II (Springer Verlag, Berlin 1975).

[14] J. M. Chambers, C. L. Mallows, B. W. Stuck, J. Am Stat. Assoc. 71, 340 (1976).

[15] T. Koren et al. (unpublished)

[16] A. Carpinteri and F. Mainardi (Eds.), Fractals and Fractional Calculus in Continuum Mechanics, CISM Courses and Lectures, Vol. 378 (Springer-Verlag, Wien, 1997).

[17] I. Eliazar and J. Klafter, Physica A 336, 219 (2004); T. Koren, A. V. Chechkin, and J. Klafter, Physica A 379, 10 (2007). 